\title{
ORIGINAL ARTICLE \\ Severe spasticity in lower extremities is associated with reduced adiposity and lower fasting plasma glucose level in persons with spinal cord injury
}

\author{
I-Y Jung ${ }^{1}, \mathrm{H}-\mathrm{R} \mathrm{Kim}^{2}$, SM Chun ${ }^{1}$ J-H Leigh ${ }^{1}$ and H-I Shin ${ }^{1}$
}

\begin{abstract}
Study design: Cross-sectional study.
Objectives: To investigate the effects of severe lower extremity spasticity on anthropometric dimensions, body composition and metabolic profiles in persons with chronic motor complete spinal cord injury (SCI).

Setting: Outpatient clinic.

Methods: Fifty-five of the 61 participants were divided into two groups (no or mild spasticity group, 28; severe spasticity group, 27) based on the assessment of the extensor muscle spasticity according to the modified Ashworth scale. Anthropometric dimensions (waist circumference (WC), waist-to-height ratio (WtHR)), body composition (fat mass (FM), body fat percentage (BFP), fat-free mass (FFM), fat-free mass percentage (FFMP), bone mineral density (BMD)) and metabolic profiles (leptin, total cholesterol, low-density lipoprotein (LDL), high-density lipoprotein (HDL), triglyceride (TG), fasting plasma glucose (FPG) and glycosylated hemoglobin (HbAlc)) were compared between the two groups with different degree of spasticity.

Results: Spasticity of the extensor muscle group negatively correlated with BFP $(r=-0.458, P<0.001)$. Patients with severe spasticity showed a lower WC and WtHR than those in the no or mild spasticity group $(P=0.038, P=0.006$, respectively). The FM, $\mathrm{BFP}$, leptin and FPG of the severe spasticity group were significantly lower than those of the patients in the no or mild spasticity group $(P=0.003, P<0.001, P<0.001$ and $P=0.037$, respectively). However, no differences in BMD, total cholesterol, LDL, HDL, TG and $\mathrm{HbAlc}$ were observed between the groups.

Conclusions: The results of this study suggest that severe spasticity in lower extremities is associated with reduced adiposity and lower FPG levels in persons with chronic motor complete SCI.
\end{abstract}

Spinal Cord (2017) 55, 378-382; doi:10.1038/sc.2016.132; published online 13 September 2016

\section{INTRODUCTION}

Spasticity is known to manifest as complex symptoms of increased skeletal muscle tone, reflex and clonus, which result from an injury to the upper motor neurons, such as spinal cord injury (SCI). ${ }^{1}$ The development of spasticity after SCI is known to impair the quality of life by restricting the patients' activities of daily living, effective walking and functional movement, as well as by inducing pain. ${ }^{2,3}$

By contrast, spasticity may have beneficial effects on paralyzed skeletal muscle, that is, defending against skeletal muscle atrophy. ${ }^{4-7}$ Gorgey and Dudley ${ }^{6}$ demonstrated that eight higher-level SCI persons showed larger thigh muscle cross-sectional areas as compared with five lower level (T12-L2) SCI persons with no or mild spasticity. Gorgey and $\mathrm{Gater}^{7}$ also showed that knee extensor spasticity positively correlated with knee extensor and whole thigh skeletal muscle cross-sectional areas.

However, with the exception of the report by Gorgey et al.,$^{5}$ few reports providing evidence that these local effects of spasticity on paralyzed muscles could have effects on the whole body composition and metabolic profiles are available. Gorgey et al. ${ }^{5}$ showed that knee extensor spasticity negatively correlated with abdominal circumference, plasma level of low-density lipoprotein (LDL) cholesterol and body fat percentage (BFP) estimated from dual-energy X-ray absorptiometry in 10 individuals with chronic motor complete SCI.

In the present study, we compared anthropometric measurements, body composition and metabolic profiles of SCI patients with severe spasticity with those of SCI patients with no or mild spasticity, while excluding patients with moderate spasticity in a larger sample compared in a previous study. ${ }^{5}$ Through these analyses, we sought to demonstrate the beneficial effects of severe spasticity on body composition and metabolic profiles in individuals with chronic motor complete SCI.

\section{PARTICIPANTS AND METHODS}

A total of 62 patients (47 men and 15 women) with chronic complete SCI who visited outpatient clinic were initially enrolled in the study. The study was approved by the Institutional Review Board of Seoul National University

${ }^{1}$ Department of Rehabilitation Medicine, Seoul National University College of Medicine, Seoul, South Korea and ${ }^{2}$ Department of Rehabilitation Medicine, National Rehabilitation Center, Seoul, South Korea

Correspondence: Professor H-I Shin, Department of Rehabilitation Medicine, Seoul National University College of Medicine, 101 Daehak-ro, Jongno-gu, Seoul 03080, Republic of Korea.

E-mail: hyungik1@snu.ac.kr

Received 29 February 2016; revised 14 July 2016; accepted 19 July 2016; published online 13 September 2016 
Hospital (IRB No. 1412-115-634); all participants read and signed a study consent form approved by the local Ethics Committee.

\section{Inclusion and exclusion criteria}

The subjects were included if they (a) were aged between 18 and 60 years; (b) had an interval of at least 1 year since the injury; (c) were motor complete SCI patients; American spinal injury Association Impairment Scale A or B; and (d) had a body mass index (BMI) between 15 and $30 \mathrm{~kg} \mathrm{~m}^{-2}$. Subjects with an BMI $<15$ or $>30 \mathrm{~kg} \mathrm{~m}^{-2}$ were excluded, as an excessively lean or obese status could mask the effects of spasticity on body composition and metabolic profiles; also, it has been reported that the dual-energy X-ray absorptiometry significantly underestimates BFP in very lean individuals, while overestimating it in very obese persons. ${ }^{8}$

\section{Evaluation of spasticity}

The modified Ashworth scale (MAS) for the knee extensors and ankle extensors was used to evaluate lower extremity spasticity. The MAS comprises 6 grades according to the severity of spasticity; we converted MAS $1+$ grade to 2. MAS grades 2,3 and 4 were changed to 3,4 and 5, respectively, as shown in Table $1 ., 9$

The MAS was measured in the supine position by the same examiner. The

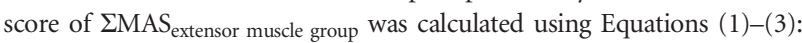

(1) $\mathrm{MAS}_{\text {ave knee extensors }}=\left(\mathrm{MAS}_{\text {right knee extensor }}+\mathrm{MAS}_{\text {left knee extensor }}\right) / 2$

(2) $\mathrm{MAS}_{\text {ave ankle extensors }}=\left(\mathrm{MAS}_{\text {right ankle extensor }}+\mathrm{MAS}_{\text {left ankle extensor }}\right) / 2$

(3) $\Sigma \mathrm{MAS}_{\text {extensor muscle group }}=\mathrm{MAS}_{\text {ave knee extensors }}+\mathrm{MAS}_{\text {ave ankle extensors }}$

The $\Sigma \mathrm{MAS}_{\text {extensor muscle group }}$ score ranges from 0 to 10 ; the subjects with a score $\leqslant 2$ were classified as having no or mild spasticity and the participants with a score $\geqslant 4$ were classified as having severe spasticity. The subjects with a score $>2$ and $<4$ were classified as having moderate spasticity and were excluded from the analysis to make clear the difference of spasticity. The Spinal Cord Assessment Tool for Spastic Reflexes (SCATS) ${ }^{10}$ and Penn Spasm Frequency Scale (PSFS) $)^{11}$ scores were also measured.

\section{Anthropometrics and dual-energy X-ray absorptiometry}

Body weight $(\mathrm{kg})$ and height $(\mathrm{m})$ were measured in each patient. The weights of the candidates were measured using a wheelchair scale (Kyongin medical, Seoul, Korea) while they sat in a wheelchair; to determine the weight of the candidates, the weight of the wheelchair was subtracted from the total weight. BMI $\left(\mathrm{kg} \mathrm{m}^{-2}\right)$ was calculated by dividing weight $(\mathrm{kg})$ by height squared $\left(\mathrm{m}^{2}\right)$; the waist-to-height ratio (WtHR) was calculated by dividing waist $(\mathrm{cm})$ by height $(\mathrm{cm})$. Waist circumference (WC) was measured at the end of normal expiration with the subject in the supine position by using a flexible tapeline at a level midway between the lowest rib and the superior border of the iliac crest; the measurement was rounded to the nearest $0.1 \mathrm{~cm} .{ }^{12}$

A dual-energy X-ray absorptiometry scanner (GE Lunar Prodigy, Madison, WI, USA, coefficient of variability $<1 \%$ ) was used to measure fat-free mass (FFM, kg), fat mass (FM, kg), fat-free mass percentage (FFMP, \%), BFP (\%) and bone mineral density (BMD, $\mathrm{g} \mathrm{cm}^{-2}$ ). With regard to $\mathrm{BMD}$, the average value of the bilateral lower extremities for one group was compared with that for the other group. The subjects were transferred from the wheelchair to an adjustable height stretcher car with the help of two persons and then moved to a scanner bed using a transfer board.

\section{Lipid and glucose metabolic profiles}

Prior to undergoing a blood examination, the patients fasted for at least 8 hours. Total cholesterol, LDL, high-density lipoprotein, triglycerides, fasting plasma glucose (FPG) and glycosylated hemoglobin (HbAlc) were assessed. The plasma concentrations of lipid profiles and glucose concentrations were determined using the commercially available chemistry analyzer AU5800 (Beckman Coulter, Brea, CA, USA) and HbAlc was checked by VARIANT II TURBO HbAlc testing system (Bio-Rad Laboratories, Hercules, CA, USA). The serum levels of leptin were assayed by radioimmunoassay using the Human Leptin RIA Kit (LINCO Research, St Charles, MO, USA). ${ }^{13}$

\section{Statistical analyses}

Spearman's rank correlation test was used to correlate lower limb spasticity and the body composition data: BFP and FFMP. A chi-square test, one-sample $t$-test and independent-sample $t$-test were used to compare the clinical and laboratory characteristics of the two groups. Statistical Package for the Social Sciences version 20.0 (IBM, Armonk, NY, USA) was used for the analysis. $P$-values $<0.05$ were considered to be statistically significant.

\section{RESULTS}

Of the 62 participants, 1 subject was excluded as insufficient information was available regarding spasticity. Moreover, six further participants were classified as having moderate spasticity and were excluded from the comparative analysis. Thus 55 subjects were finally included in the analysis (Figure 1). Of these patients, 43 were male and 12 were female. The mean age of the patients was $42.4 \pm 9.6$ years, and the mean period of injury was $14.3 \pm 8.5$ years. The average BMI and WC were $21.9 \pm 2.9 \mathrm{~kg} \mathrm{~m}^{-2}$ and $85.6 \pm 10.4 \mathrm{~cm}$, respectively. Of the 55 patients with SCI, 35 had a cervical lesion and 20 had a thoracic lesion. According to the American spinal injury Association Impairment Scale, 31 patients were classified as A and 24 were classified as B.

Correlation between lower limb spasticity and body composition Spearman's rank correlation test was used to correlate $\Sigma \mathrm{MAS}_{\text {extensor muscle group }}$ to BFP and FFMP. The $\Sigma \mathrm{MAS}_{\text {extensor muscle }}$ group negatively correlated with BFP (correlation coefficient $=-0.458$, $P<0.001$, Figure 2). The $\Sigma \mathrm{MAS}_{\text {extensor muscle group positively correlated }}$ with FFMP (correlation coefficient $=0.441, P<0.001$ ).

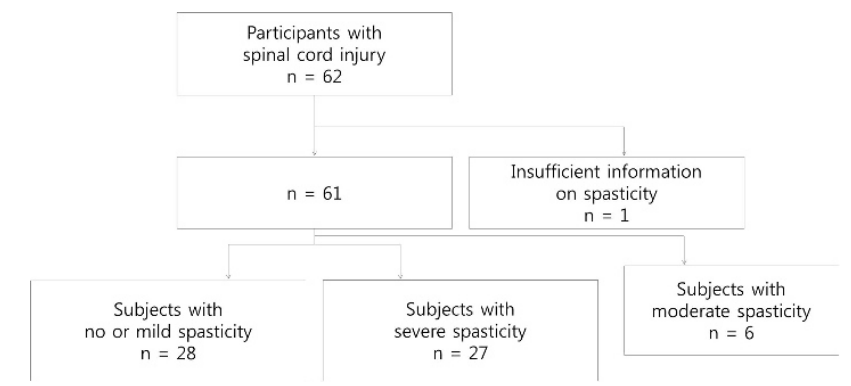

Figure 1 Flow diagram of grouping the subjects according to the severity of spasticity.

\section{Table 1 Six grades of the modified Ashworth scale}

Grade 0: No increase in muscle tone

Grade 1: Slight increase in muscle tone, manifested by a catch or by minimal resistance at the end of the ROM, when the affected part(s) is moved in flexion or extension Grade 2: Slight increase in muscle tone, manifested by a catch, followed by minimal resistance throughout the remainder (less than half) of the ROM

Grade 3: More marked increase in muscle tone through most of the ROM, but the affected part(s) can be easily moved

Grade 4: Considerable increase in muscle tone, and passive movement is difficult

Grade 5: Affected part(s) is rigid in flexion or extension

Abbreviation: ROM, range of motion. 


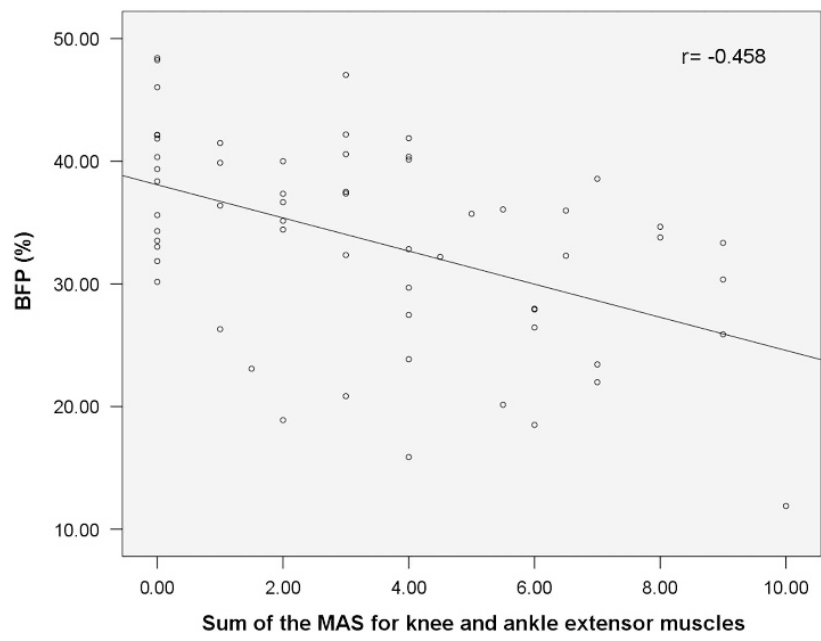

Figure 2 Correlation between the sum of the modified Ashworth scale (MAS) for knee and ankle extensor muscles and body fat percentage (BFP).

\section{Comparisons between the groups according to the severity of spasticity}

Gender, level of injury, age, BMI and duration of injury did not differ between the two groups (Table 2). Based on the

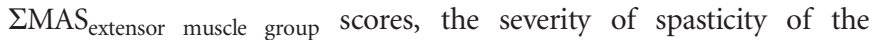
extensor muscle groups significantly differed between the two groups $(P<0.001)$. Moreover, the SCATS and PSFS scores also differed between the two groups.

\section{Anthropometric measurements and body composition}

The subjects in the severe spasticity group had a smaller WC $(P=0.038)$ and lower WtHR $(P=0.006)$ as compared with those in the no or mild spasticity group. Moreover, FM and BFP were lower in the severe spasticity group than in the no or mild spasticity group $(P=0.003$ and $P<0.001$, respectively). FFMP was higher in the severe spasticity group than in the no or mild spasticity group $(P<0.001)$. However, BMD values did not differ between the two groups (see Table 3). No significant differences between paraplegic and tetraplegic subjects in FM, FFM, BFP and FFMP were observed (the data are not shown).

\section{Metabolic profiles}

In the severe spasticity group, the serum leptin levels were evaluated in 25 subjects, but all other metabolic profiles were evaluated in 27 subjects. The serum leptin and FPG levels were lower in the severe spasticity group than in the no or mild spasticity group $(P<0.001$ and $P=0.037$, respectively). However, no difference in the lipid profile and HbAlc levels was observed between the two groups (see Table 4).

\section{DISCUSSION}

Compared with the chronic spinal cord injury patients with no or mild spasticity, those with severe spasticity showed reduced adiposity: lower BFP and FM. The sum of MAS for knee extensor muscles and ankle extensor muscles negatively correlated with BFP. These results are compatible with previous results that showed the beneficial effects of spasticity on whole body composition. ${ }^{5}$ More importantly, WC, which is known to be a good surrogate measure of visceral adipose tissue in the persons with SCI, as well as in able-bodied population, was lower in the severe spasticity group. ${ }^{14,15}$ Because increase in visceral adipose tissue is known to be associated with altered
Table 2 Comparisons between groups according to the severity of spasticity

\begin{tabular}{|c|c|c|}
\hline & $\begin{array}{l}\text { No or mild spasticity } \\
\qquad(\mathrm{n}=28)\end{array}$ & $\begin{array}{l}\text { Severe spasticity } \\
\qquad(\mathrm{n}=27)\end{array}$ \\
\hline Sex (male/female) & $20 / 8$ & $23 / 4$ \\
\hline $\begin{array}{l}\text { Level of injury (cervical: } \\
\text { thoracic) }\end{array}$ & $15: 13$ & $20: 7$ \\
\hline Age (years) & $44.6 \pm 9.8$ & $40.0 \pm 8.9$ \\
\hline $\mathrm{BMI}\left(\mathrm{kg} \mathrm{m}^{-2}\right)$ & $22.4 \pm 2.7$ & $21.5 \pm 3.1$ \\
\hline $\begin{array}{l}\text { Mean duration of injury } \\
\text { (years) }\end{array}$ & $12.4 \pm 8.1$ & $16.4 \pm 8.6$ \\
\hline $\begin{array}{l}\Sigma \text { MAS score: extensor } \\
\text { muscles* }\end{array}$ & $0.84 \pm 1.03$ & $6.04 \pm 1.82$ \\
\hline SCATS: ankle clonus* & $1.00 \pm 1.19$ & $2.02 \pm 1.08$ \\
\hline SCATS: flexor spasm* & $0.30 \pm 0.50$ & $1.22 \pm 1.01$ \\
\hline SCATS: extensor spasm* & $0.36 \pm 0.47$ & $1.56 \pm 0.97$ \\
\hline PSFS: frequency* & $1.00 \pm 1.02$ & $1.93 \pm 1.17$ \\
\hline PSFS: severity* & $1.07 \pm 1.02$ & $2.19 \pm 0.79$ \\
\hline
\end{tabular}

Abbreviations: BMI, body mass index; MAS, modified Ashworth scale; PSFS, Penn Spasm Frequency Scale; SCATS, Spinal Cord Assessment Tool for Spastic Reflexes. ${ }^{*} P<0.001$.

carbohydrate and lipid metabolism in both able-bodied and SCI populations, ${ }^{16,17}$ severe spasticity, especially in lower extremities as presented in this study, might be regarded as to have protective effects against adverse metabolic profiles commonly seen in individuals with SCI.

However, the lipid profiles, including LDL cholesterol level, did not differ between the two groups in the present study. This could be regarded as inconsistent with a previous report that demonstrated a negative correlation between knee extensor spasticity and plasma LDL cholesterol level. ${ }^{5}$ Considering that several variables, such as genetics, diet, exercise and smoking, can influence blood profile measurements, a difference in the local skeletal muscle activity owing to spasticity may not lead to a significant difference in the lipid profile. Similarly, the effects of functional electrical stimulation applied to paralyzed muscles on lipid profiles remain debatable, as some trials have shown a modest improvement in lipid panels, whereas others have documented no changes. ${ }^{18,19}$

In the present study, the FPG level was found to be lower in the severe spasticity group. This result could be partially explained by the higher glucose uptake in more spastic muscles. ${ }^{4}$ However, as neither oral glucose tolerance nor fasting or stimulated plasma insulin concentrations were evaluated in our study, a possible difference in insulin sensitivity between the groups discordant for the level of spasticity could not be addressed. In a previous study, spasticity did not influence insulin sensitivity, which was estimated from surrogate markers, such as Matsuda and Defronzo formula or the homeostasis model assessment index, although an increase in hip extensor spasticity correlated with a reduction in plasma insulin concentration at T30 (30 min after oral glucose administration). ${ }^{5}$

In addition, the BMD did not differ between the two groups. The results of previous studies on the influence of spasticity on bone density have been inconsistent, and attempts to improve bone mass by modulating muscle tone, activity or weight bearing have not yielded clear benefits. ${ }^{20,21}$

In the SCI persons with severe spasticity, recruitment of large skeletal muscle contraction by involuntary spasticity could result in higher energy expenditure and defend against the BFP increment. Additionally, researchers also suggested several hypotheses behind the 
Table 3 Anthropometric measurements and body composition

\begin{tabular}{lcc}
\hline & No or mild spasticity $(\mathrm{n}=28)$ & Severe spasticity $(\mathrm{n}=27)$ \\
\hline $\mathrm{WC}(\mathrm{cm})^{* *}$ & $88.42 \pm 10.58$ & $82.63 \pm 9.53$ \\
$\mathrm{WtHR}^{*}$ & $0.53 \pm 0.06$ & $0.48 \pm 0.05$ \\
$\mathrm{FM}(\mathrm{kg})^{*}$ & $24.46 \pm 6.74$ & $18.77 \pm 6.55$ \\
$\mathrm{BFP}(\%)^{*}$ & $37.12 \pm 6.24$ & $29.60 \pm 7.69$ \\
FFM (kg) & $41.23 \pm 8.73$ & $43.65 \pm 7.96$ \\
FFMP $(\%)^{*}$ & $62.88 \pm 6.24$ & $70.40 \pm 7.69$ \\
BMD $\left(\mathrm{g} \mathrm{cm}^{-2}\right)$ & $1.09 \pm 0.11$ & $1.06 \pm 0.09$ \\
\hline
\end{tabular}

Abbreviations: BFP, body fat percentage; BMD, bone mineral density; FM, fat mass; FFM, fatfree mass; FFMP, fat-free mass percentage; WC, waist circumference; WtHR, waist-to-height ratio. ${ }^{*} P<0.01, * * P<0.05$

protective effects of spasticity. First, spastic muscles might attenuate the shift in slow-to-fast muscle fiber transformation; slow fibers have a higher mitochondrial concentration that may be responsible for the increased fat oxidation. ${ }^{22,23}$ Second, SCI is characterized by diminished growth hormone $(\mathrm{GH})$, insulin-like growth factor-1 (IGF-1) and testosterone; the decline in anabolic hormones may further exacerbate muscle atrophy and FM gain below the level of injury. ${ }^{24-26}$ Spasticity may provoke muscular IGF-1 that compensates for the reduced growth hormone-IGF-1 axis after SCI. ${ }^{7}$

Although MAS is the most commonly used tool for measuring spasticity in clinical practice and research, the reliability and validity of this scale remain unclear. ${ }^{27,28}$ Moreover, the degree of spasticity can vary according to the patient's physical and emotional conditions, even within a single day. ${ }^{28}$ Therefore, to separate the two groups (no or mild spasticity group vs severe spasticity group) clearly, we excluded six subjects with moderate spasticity from the analysis. In addition, to ensure an accurate assessment of the differences between the groups, we evaluated the SCATS and the PSFS scores, as well as the MAS scores. The SCATS measures spastic reflexes of individuals with SCI. This scale is divided into three subscales, each addressing a separate spasm: clonus, flexor spasms, and extensor spasms. To assess each subscale, a spasm is triggered and then rated with a score ranging from 0 to $3 .{ }^{10}$ On the other hand, the PSFS is a self-reported measure that assesses an individual's perception of spasticity frequency and severity. ${ }^{11}$ Both SCATS and PSFS scores were much higher in the severe spasticity group than in the no or mild spasticity group.

This study has several limitations. First, the medications administered to alleviate spasticity may cause increased FM and altered glucose homeostasis. On the contrary, some authors reported that the administration of low-dose oral baclofen resulted in an increased IGF-1, which is known to increase FFM and reduce FM. ${ }^{29}$ However, we did not collect information regarding the medications administered to the participants and, therefore, we were unable to estimate the influence of medications, such as baclofen, on our results. Second, to verify the beneficial effects of severe spasticity on cardiovascular risk modulation in persons with SCI, its impact on insulin sensitivity should be explored. However, we did not measure insulin concentration, and therefore, we were not able to demonstrate the difference in insulin sensitivity between the two groups.

For future research, it would be necessary to explore the insulin sensitivity in SCI persons with severe spasticity, which could be estimated from plasma insulin and glucose concentration before and after oral glucose administration. In addition, mechanisms through which severe spasticity ameliorates the derangement of body compositions and glucose homeostasis could be investigated. For example, differences in the expression of glucose transport protein,
Table 4 Comparison of metabolic profiles between the no or mild and severe spasticity groups

\begin{tabular}{lcc}
\hline & No or mild spasticity $(\mathrm{n}=28)$ & Severe spasticity $(\mathrm{n}=27)$ \\
\hline Leptin $\left(\mathrm{ng} \mathrm{ml^{-1 }}\right)$ & $16.33 \pm 9.62$ & $7.64 \pm 4.38$ \\
Total cholesterol & $186.96 \pm 29.11$ & $180.04 \pm 30.15$ \\
$\left.(\mathrm{mg} \mathrm{dl})^{-1}\right)$ & & \\
$\mathrm{LDL}\left(\mathrm{mg} \mathrm{dl}^{-1}\right)$ & $117.96 \pm 25.60$ & $111.07 \pm 23.38$ \\
$\mathrm{HDL}\left(\mathrm{mg} \mathrm{dl}^{-1}\right)$ & $41.04 \pm 9.36$ & $41.82 \pm 7.39$ \\
$\mathrm{TG}\left(\mathrm{mg} \mathrm{dl}^{-1}\right)$ & $166.64 \pm 90.67$ & $130.85 \pm 70.94$ \\
$\mathrm{FPG}\left(\mathrm{mg} \mathrm{dl}^{-1}\right)^{* *}$ & $97.93 \pm 18.97$ & $89.37 \pm 8.50$ \\
$\mathrm{HbAlc}(\%)$ & $5.30 \pm 0.56$ & $5.17 \pm 0.64$ \\
\hline
\end{tabular}

Abbreviations: FPG, fasting plasma glucose; HbAlc, glycosylated hemoglobin; HDL, high-density lipoprotein; LDL, low-density lipoprotein; TG, triglycerides. ${ }^{*} P<0.01$, ${ }^{* *} P<0.05$.

GLUT4 proteins, ${ }^{30}$ IGF-1 concentration ${ }^{7,29}$ and muscle fiber type composition $^{22,23}$ have been suggested as related mechanisms.

This study suggests that, in individuals with SCI, severe spasticity in lower limbs could influence the body composition and glucose homeostasis. Hence, severe spasticity might be included as an associated variable in future studies on cardiovascular risk factors in individuals with SCI.

\section{DATA ARCHIVING}

There were no data to deposit.

\section{CONFLICT OF INTEREST}

The authors declare no conflict of interest.

\section{ACKNOWLEDGEMENTS}

This study was supported by the Bio \& Medical Technology Development Program of the National Research Foundation (NRF) of Korea funded by the Ministry of Science, Information and Communication Technology (ICT) \& Future Planning (grant no. 2013R1A1A2010246).

1 Pandyan AD, Gregoric M, Barnes MP, Wood D, Van Wijck F, Burridge J et al. Spasticity: clinical perceptions, neurological realities and meaningful measurement. Disabil Rehabil 2005; 27: 2-6.

2 Adams MM, Hicks AL. Spasticity after spinal cord injury. Spinal Cord 2005; 43 577-586.

3 Sköld C, Levi R, Seiger A. Spasticity after traumatic spinal cord injury: nature, severity, and location. Arch Phys Med Rehabil 1999; 80: 1548-1557.

4 Bennegard GM, Karlsson AK. Higher glucose uptake in paralysed spastic leg. Spinal Cord 2008; 46: 103-106.

5 Gorgey AS, Chiodo AE, Zemper ED, Hornyak JE, Rodriguez GM, Gater DR et al. Relationship of spasticity to soft tissue body composition and the metabolic profile in persons with chronic motor complete spinal cord injury. J Spinal Cord Med 2010; 33: 6-15.

6 Gorgey AS, Dudley GA. Spasticity may defend skeletal muscle size and composition after incomplete spinal cord injury. Spinal Cord 2008; 46: 96-102.

7 Gorgey AS, Gater DR. Insulin growth factors may explain relationship between spasticity and skeletal muscle size in men with spinal cord injury. J Rehabil Res Dev 2012; 49: 373-380.

8 LaForgia J, Dollman J, Dale MJ, Withers RT, Hill AM. Validation of DXA body composition estimates in obese men and women. Obesity 2009; 17: 821-826.

9 Bohannon RW, Smith MB. Interrater reliability of a modified Ashworth scale of muscle spasticity. Phys Ther 1987; 67: 206-207.

10 Benz EN, Hornby TG, Bode RK, Scheidt RA, Schmit BD. A physiologically based clinical measure for spastic reflexes in spinal cord injury. Arch Phys Med Rehabil 2005; 86: 52-59.

11 Adams MM, Ginis KA, Hicks AL. The spinal cord injury spasticity evaluation tool: development and evaluation. Arch Phys Med Rehabil 2007; 88: 1185-1192.

12 Alberti KG, Zimmet P, Shaw J. Metabolic syndrome-a new world-wide definition. A consensus Statement from the International Diabetes Federation. Diabet Med 2006; 23: $469-480$

$13 \mathrm{Ma} \mathrm{Z}$, Gingerich RL, Santiago JV. Radioimmunoassay of leptin in human plasma. Clin Chem 1996; 42: 942-946. 
14 Edwards LA, Bugaresti JM, Buchholz AC. Visceral adipose tissue and the ratio of visceral to subcutaneous adipose tissue are greater in adults with than in those without spinal cord injury, despite matching waist circumferences. Am J Clin Nutr 2008; 87: 600-607.

15 Pouliot MC, Després JP, Lemieux S, Moorjani S, Bouchard C, Tremblay A et al. Waist circumference and abdominal sagittal diameter: best simple anthropometric indexes of abdominal visceral adipose tissue accumulation and related cardiovascular risk in men and women. Am J Cardiol 1994; 73: 460-468.

16 Fujioka S, Matsuzawa Y, Tokunaga K, Tarui S. Contribution of intra-abdominal fat accumulation to the impairment of glucose and lipid metabolism in human obesity. Metabolism 1987; 36: 54-59.

17 Gorgey AS, Mather KJ, Gater DR. Central adiposity associations to carbohydrate and lipid metabolism in individuals with complete motor spinal cord injury. Metabolism 2011; 60: 843-851.

18 Gorgey AS, Martin H, Metz A, Khalil RE, Dolbow DR, Gater DR. Longitudinal changes in body composition and metabolic profile between exercise clinical trials in men with chronic spinal cord injury. J Spinal Cord Med 2016; 17: 1-14.

19 Solomonow M, Reisin E, Aguilar E, Baratta RV, Best R, D'Ambrosia R. Reciprocating gait orthosis powered with electrical muscle stimulation (RGO II). Part II: Medical evaluation of 70 paraplegic patients. Orthopedics 1997; 20: 411-418.

20 Johnston TE, Marino RJ, Oleson CV, Schmidt-Read M, Leiby BE, Sendecki J. Musculoskeletal effects of 2 functional electrical stimulation cycling paradigms conducted at different cadences for people with spinal cord injury: a pilot study. Arch Phys Med Rehabil 2015; 97: 1413-1422.

21 Löfvenmark I, Werhagen L, Norrbrink C. Spasticity and bone density after a spinal cord injury. J Rehabil Med 2009; 41: 1080-1084.
22 Harris RL, Bobet J, Sanelli L, Bennett DJ. Tail muscles become slow but fatigable in chronic sacral spinal rats with spasticity. J Neurophysiol 2006; 95: 1124-1133.

23 Harris RL, Putman CT, Rank M, Sanelli L, Bennett DJ. Spastic tail muscles recover from myofiber atrophy and myosin heavy chain transformations in chronic spinal rats. J Neurophysiol 2007; 97: 1040-1051.

24 Bauman WA, Spungen AM, Flanagan S, Zhong YG, Alexander LR, Tsitouras PD. Blunted growth hormone response to intravenous arginine in subjects with a spinal cord injury. Horm Metab Res 1994; 26: 152-156.

25 Halstead LS, Groah SL, Libin A, Hamm LF, Priestley L. The effects of an anabolic agent on body composition and pulmonary function in tetraplegia: a pilot study. Spinal Cord 2010; 48: 55-59.

26 Gregory CM, Vandenborne K, Huang HF, Ottenweller JE, Dudley GA. Effects of testosterone replacement therapy on skeletal muscle after spinal cord injury. Spinal Cord 2003; 41: 23-28.

27 Pandyan AD, Johnson GR, Price Cl, Curless RH, Barnes MP, Rodgers H. A review of the properties and limitations of the Ashworth and modified Ashworth scales as measures of spasticity. Clin Rehabil 1999; 13: 373-383.

28 Sköld C. Spasticity in spinal cord injury: self- and clinically rated intrinsic fluctuations and intervention-induced changes. Arch Phys Med Rehabil 2000; 81: 144-149.

29 Bauman WA, La Fountaine MF, Cirnigliaro CM, Kirshblum SC, Spungen AM. Low-dose baclofen therapy raised plasma insulin-like growth factor- 1 concentrations, but not into the normal range in a predictable and sustained manner in men with chronic spinal cord injury. J Spinal Cord Med 2013; 36: 476-482.

30 Biens $\varnothing$ RS, Ringholm S, Kiilerich K, Aachmann-Andersen NJ, Krogh-Madsen R, Guerra B et al. GLUT4 and glycogen synthase are key players in bed rest-induced insulin resistance. Diabetes 2012; 61: 1090-1099. 